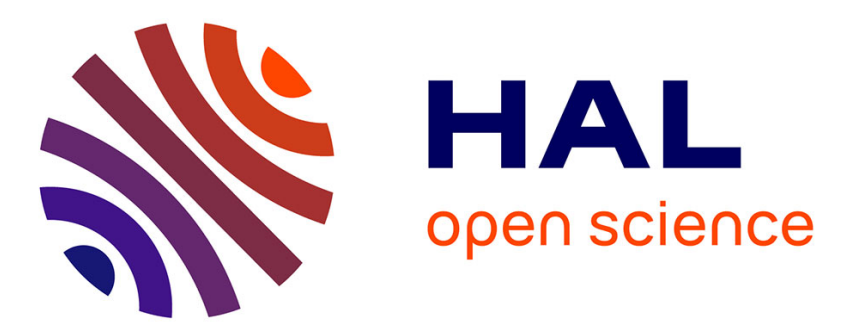

\title{
A Gradient Scheme for the Discretization of Richards Equation
}

\author{
Konstantin Brenner, Danielle Hilhorst, Huy-Cuong Vu-Do
}

\section{To cite this version:}

Konstantin Brenner, Danielle Hilhorst, Huy-Cuong Vu-Do. A Gradient Scheme for the Discretization of Richards Equation. The International Symposium of Finite Volumes for Complex Applications VII, Jun 2014, Berlin, Germany. 10.1007/978-3-319-05591-6_53 . hal-01317588

\section{HAL Id: hal-01317588 \\ https://hal.science/hal-01317588}

Submitted on 18 May 2016

HAL is a multi-disciplinary open access archive for the deposit and dissemination of scientific research documents, whether they are published or not. The documents may come from teaching and research institutions in France or abroad, or from public or private research centers.
L'archive ouverte pluridisciplinaire HAL, est destinée au dépôt et à la diffusion de documents scientifiques de niveau recherche, publiés ou non, émanant des établissements d'enseignement et de recherche français ou étrangers, des laboratoires publics ou privés. 


\title{
A Gradient scheme for the discretization of Richards Equation
}

\author{
Konstantin Brenner, Danielle Hilhorst, Huy Cuong Vu Do
}

\begin{abstract}
We propose a finite volume method on general meshes for the discretization of Richards equation, an elliptic - parabolic equation modeling groundwater flow. The diffusion term, which can be anisotropic and heterogeneous, is discretized in a gradient scheme framework, which can be applied to a wide range of unstructured possibly non-matching polyhedral meshes in arbitrary space dimension. More precisely, we implement the SUSHI scheme which is also locally conservative. As is needed for Richards equation, the time discretization is fully implicit. We obtain a convergence result based upon energy-type estimates and the application of the Fréchet-Kolmogorov compactness theorem. We implement the scheme and present the results of a number of numerical tests.
\end{abstract}

\section{Richards equation}

In this article, we study Richards equation using Kirchhoff transformation. Let $\Omega$ be a open bounded polygonal subset of $\mathbb{R}^{d}(d=1,2$ or 3$)$ and let $T$ be a positive real number; Richards equation in the space-time domain $Q_{T}=\Omega \times(0, T)$ is given by

$$
\partial_{t}(\phi(\mathbf{x}) \theta(p))-\operatorname{div}\left(k_{r}(\theta(p)) \mathbf{K}(\mathbf{x}) \nabla(p+z)\right)=0,
$$

where $p(\mathbf{x}, t)$ is pressure head. The function $\theta(p)$ is the water saturation, $\phi(\mathbf{x})$ is the porosity, $\mathbf{K}(\mathbf{x})$ is the absolute permeability tensor and the scalar function $k_{r}(\boldsymbol{\theta})$

Konstantin Brenner, LJAD University Nice Sophia-Antipolis \& Coffee team Inria Sophia-Antipolis - Méditerranée, France, e-mail: konstantin.brenner@unice.fr

Danielle Hilhorst, Laboratoire de Mathématiques, CNRS et Université de Paris-Sud, Orsay, France, e-mail: Danielle.Hilhorst@math.u-psud.fr,

Huy Cuong Vu Do, Laboratoire de Mathématiques, Université de Paris-Sud, Orsay, France, e-mail: vdhuycuong@math.u-psud.fr,

D. Hilhorst and H.C. Vu Do acknowledge the support of the ITN Marie Curie Project FIRST and of the Fondation Jacques Hadamard 
corresponds to the relative permeability, which depends on the water content. The space coordinates are defined by $\mathbf{x}=(x, z)$ in the case of space dimension 2 and $\mathbf{x}=(x, y, z)$ in the case of space dimension 3 . Next we perform Kirchhoff's transformation. We set

$$
F(s):=\int_{0}^{s} k_{r}(\theta(\tau)) d \tau
$$

and suppose that the function $F$ is invertible. Then we set $u=F(p)$ in $Q_{T}$ and $c(u)=c(F(p))=\theta(p)$. We remark that Kirchhoff's transformation leads to $\nabla u=$ $k_{r}(\theta(p)) \nabla p$. Thus, the equation (1) becomes

$$
\partial_{t}(\phi(\mathbf{x}) c(u))-\operatorname{div}(\mathbf{K}(\mathbf{x}) \nabla u)-\operatorname{div}\left(k_{r}(c(u)) \mathbf{K}(\mathbf{x}) \nabla z\right)=0 .
$$

Next, we consider the equation (2) together with the inhomogeneous Dirichlet boundary and the initial conditions

$$
\begin{array}{ll}
u(\mathbf{x}, t)=\hat{u}(\mathbf{x}) & \text { a.e. on } \partial \Omega \times(0, T), \\
u(\mathbf{x}, 0)=u_{0}(\mathbf{x}) & \text { a.e. in } \Omega .
\end{array}
$$

We make the following hypotheses:

$\left(H_{1}\right) c$ is a continuous nondecreasing function such that there $\bar{\xi}>0$ and $\underline{\xi} \geq 0$ satisfying $|c(u)| \leq \bar{\xi}(1+|u|)$ for all $u \in \mathbb{R}$ and $|c(u)-c(v)| \geq \underline{\xi}|u-v|$ for all $u, v \in \mathbb{R}$. $\left(H_{2}\right) k_{r}$ is a continuous function such that $0 \leq k_{r} \leq \overline{k_{r}}$.

$\left(H_{3}\right) \mathbf{K}$ is a bounded function from $\Omega$ to $\mathbb{M}_{d}(\mathbb{R})$, where $\mathbb{M}_{d}(\mathbb{R})$ denotes the set of real $d \times d$ matrices. Moreover for a.e. $\mathbf{x}$ in $\Omega, \mathbf{K}(\mathbf{x})$ is a symmetric positive definite matrix and there exist two positive constants $\overline{\mathrm{K}}$ and $\underline{\mathrm{K}}$ such that the eigenvalues of $\mathbf{K}(\mathbf{x})$ are included in $[\overline{\mathrm{K}}, \underline{\mathrm{K}}]$.

$\left(H_{4}\right) u_{0} \in L^{2}(\Omega), \hat{u} \in H^{1}(\Omega)$ and $\phi \in L^{\infty}(\Omega)$ is such that $0<\underline{\phi} \leq \phi(\mathbf{x}) \leq \bar{\phi}$ for a.e. $\mathbf{x} \in \Omega$.

Definition. A function $u(\mathbf{x}, t)$ is said to be a weak solution of Problem (2) - (3) if:

(i) $u(\mathbf{x}, t)-\hat{u}(\mathbf{x}) \in L^{2}\left(0, T ; H_{0}^{1}(\Omega)\right)$,

(ii) $c(u) \in L^{\infty}\left(0, T ; L^{2}(\Omega)\right)$,

(iii) $-\int_{0}^{T} \int_{\Omega} \phi(\mathbf{x}) c(u(\mathbf{x}, t)) \partial_{t} \varphi(\mathbf{x}, t) d \mathbf{x} d t-\int_{\Omega} \phi(\mathbf{x}) c\left(u_{0}(\mathbf{x})\right) \varphi(\mathbf{x}, 0) d \mathbf{x}$

$$
\begin{aligned}
& +\int_{0}^{T} \int_{\Omega} \mathbf{K}(\mathbf{x}) \nabla u(\mathbf{x}, t) \cdot \nabla \varphi(\mathbf{x}, t) d \mathbf{x} d t \\
& +\int_{0}^{T} \int_{\Omega} k_{r}(c(u(\mathbf{x}, t)) \mathbf{K}(\mathbf{x}) \nabla z \cdot \nabla \varphi(\mathbf{x}, t) d \mathbf{x} d t=0,
\end{aligned}
$$

for all $\varphi \in L^{2}\left(0, T ; H_{0}^{1}(\Omega)\right)$ with $\varphi(\cdot, T)=0$ and $\partial_{t} \varphi \in L^{\infty}\left(Q_{T}\right)$.

The discretization of Richards equation by means of gradient schemes has already been proposed by Eymard, Guichard, Herbin and Masson [3], where they consider Richards equation as a special case of two phase flow; however, they make the extra hypothesis that the relative permeability $k_{r}$ is bounded away from zero. 


\section{Gradient discretization}

Following [2] we define a gradient discretization $D$ of Problem (2) - (3) on a vector space $X_{D}$, or more precisely its subspace $X_{D}^{0}$ associated with the homogeneous Dirichlet boundary condition, and the two following linear operators:

- A gradient operator on the matrix domain: $\nabla_{D}: X_{D} \rightarrow L^{2}(\Omega)^{d}$.

- A function reconstruction operator on the matrix domain: $\pi_{D}: X_{D} \rightarrow L^{2}(\Omega)$.

Coercivity: We assume that $\left\|\nabla_{D} \cdot\right\|_{L^{2}(\Omega)^{d}}$ defines a norm on $X_{D}^{0}$. A gradient discretization $D$ is said to be coercive if there exists $C_{D} \geq 0$ such that for all $v \in X_{D}^{0}$ one has

$$
\left\|\pi_{D} v\right\|_{L^{2}(\Omega)} \leq C_{D}\left\|\nabla_{D} v\right\|_{L^{2}(\Omega)^{d}} .
$$

Consistency: Let $u \in H_{0}^{1}(\Omega)$, and let us define

$$
S_{D}(u)=\inf _{v \in X_{D}^{0}}\left(\left\|\nabla_{D} v-\nabla u\right\|_{L^{2}(\Omega)^{d}}+\left\|\pi_{D} v-u\right\|_{L^{2}(\Omega)}\right) .
$$

Then, a sequence of gradient discretizations $\left(D^{(m)}\right)_{m \in \mathbb{N}}$ is said to be consistent if for all $u \in H_{0}^{1}(\Omega), \lim _{m \rightarrow+\infty} S_{D^{(m)}}(u)=0$.

Limit Conformity: For all $\mathbf{q} \in H_{d i v}(\Omega)$, we define

$$
W_{D}(\mathbf{q})=\sup _{0 \neq v \in X_{D}^{0}} \frac{1}{\left\|\nabla_{D} v\right\|_{L^{2}(\Omega)^{d}}} \int_{\Omega} \nabla_{D^{v}} \cdot \mathbf{q}+\pi_{D^{v}} \operatorname{div}(\mathbf{q}) d \mathbf{x}
$$

Then, a sequence of gradient discretizations $\left(D^{(m)}\right)_{m \in \mathbb{N}}$ is said to be limit conforming if for all $\mathbf{q} \in H_{\text {div }}(\Omega), \lim _{m \rightarrow+\infty} W_{D^{(m)}}(\mathbf{q})=0$.

Compactness: A sequence of gradient discretizations $\left(D^{(m)}\right)_{m \in \mathbb{N}}$ is said to be compact if for all sequences $v_{m} \in X_{D^{(m)}}^{0}, m \in \mathbb{N}$ such that there exists $C>0$ with $\left\|\nabla_{D^{(m)}} v_{m}\right\|_{L^{2}(\Omega)^{d}} \leq C$ for all $m \in \mathbb{N}$, then there exist $\bar{v} \in L^{2}(\Omega)$ such that

$$
\lim _{m \rightarrow+\infty}\left\|\pi_{D^{(m)}} v_{m}-\bar{v}\right\|_{L^{2}(\Omega)}=0
$$

For $N \in \mathbb{N}^{*}$, let us consider the time discretization $t^{0}=0<t^{1}<\cdots<t^{n-1}<t^{n} \cdots<$ $t^{N}=T$ of the time interval $[0, T]$. We denote the time steps by $\delta t^{n}=t^{n}-t^{n-1}$ for all $n \in\{1, \cdots, N\}$ while $\delta t$ stands for the whole sequence $\left(\delta t^{n}\right)_{n \in\{1, \ldots, N\}}$. For all $v=\left(v^{n} \in X_{D}\right)_{n=1, \cdots, N}$ we set $\pi_{D, \delta t} v(\mathbf{x}, t)=\pi_{D} v^{n}(\mathbf{x})$ and $\nabla_{D, \delta t} v(\mathbf{x}, t)=\nabla_{D} v^{n}(\mathbf{x})$ for all $(\mathbf{x}, t) \in \Omega \times\left(t^{n-1}, t^{n}\right], n \in\{1, \ldots, N\}$.

Discrete variational formulation: For a given $u^{0}, \hat{u}_{D} \in X_{D}$ find $u=\left(u^{n} \in\right.$ $\left.X_{D}\right)_{n \in\{1, \ldots, N\}}$ such that for each $n \in\{1, \ldots, N\}, u^{n}-\hat{u}_{D} \in X_{D}^{0}$ and for all $v \in X_{D}^{0}$ 


$$
\int_{\Omega} \phi \frac{c\left(\pi_{D} u^{n}\right)-c\left(\pi_{D} u^{n-1}\right)}{\delta t^{n}} \pi_{D} v d \mathbf{x}+\int_{\Omega} \mathbf{K}\left(\nabla_{D} u^{n}+k_{r}\left(\pi_{D} u^{n}\right) \nabla z\right) \cdot \nabla_{D} v d \mathbf{x}=0
$$

Proposition 1 There exists at least one solution of (6); moreover there exists a positive $C$ only depending on $\phi, \bar{\phi}, \underline{\xi}, \bar{\xi}, \underline{K}, \bar{K}, \overline{k_{r}}, \Omega, T, u_{0}$, $\hat{u}$ as well as on $\left\|c\left(\pi_{D} u^{0}\right)-c\left(u_{0}\right)\right\|_{L^{2}(\Omega)}, \| \pi_{D} \hat{u}_{D}-\overline{\hat{u}}_{L^{2}(\Omega)}$ and $\left\|\nabla_{D} \hat{u}_{D}-\nabla \hat{u}\right\|_{L^{2}(\Omega)}$ such that

$$
\left\|c\left(\pi_{D, \delta t} u\right)\right\|_{L^{\infty}\left(0, T ; L^{2}(\Omega)\right)}+\left\|\nabla_{D, \delta t} u\right\|_{L^{2}\left(Q_{T}\right)^{d}} \leq C
$$

for any solution $u$ of (6).

Proof. In order to keep this presentation short, we only prove below the priori estimate (7), and only in the case of homogeneous Dirichlet boundary conditions; the adaptation to the inhomogeneous case is straightforward, and the existence of a discrete solution can be deduced using a standard argument based upon the topological degree. Let $u=\left(u^{n}\right)_{n \in\{1, \ldots, N\}}$ be a solution of (6) and define

$$
\begin{aligned}
& A_{D, \delta t}^{n}(v)=\int_{\Omega} \phi \frac{c\left(\pi_{D} u^{n}\right)-c\left(\pi_{D} u^{n-1}\right)}{\delta t^{n}} \pi_{D} v d \mathbf{x}, \\
& B_{D, \delta t}^{n}(v)=\int_{\Omega} \mathbf{K} \nabla_{D} u^{n} \cdot \nabla_{D} v d \mathbf{x}, \quad C_{D, \delta t}^{n}(v)=\int_{\Omega} \mathbf{K} k_{r}\left(\pi_{D} u^{n}\right) \nabla_{z} \cdot \nabla_{D} v d \mathbf{x},
\end{aligned}
$$

for all $n \in\{1, \ldots, N\}$ and $v \in X_{D}^{0}$. The terms defined above satisfy

$$
A_{D, \delta t}^{n}(v)+B_{D, \delta t}^{n}(v)+C_{D, \delta t}^{n}(v)=0 \text { for all } v \in X_{D}^{0} .
$$

Let us first estimate $\sum_{n=1}^{m} \delta t^{n} A_{D, \delta t}^{n}\left(u^{n}\right)$ for $m \in\{1, \ldots, N\}$; we define

$$
\xi(u)=c(u) u-\int_{0}^{u} c(\tau) d \tau \quad \text { for all } u \in \mathbb{R} .
$$

For all $a, b \in \mathbb{R}$, one has $\xi(a)-\xi(b)=(c(a)-c(b)) a-\int_{b}^{a}(c(\tau)-c(b)) d \tau$ and since $c$ is nondecreasing we have that $\xi(a)-\xi(b) \leq(c(a)-c(b)) a$. It implies that

$$
\sum_{n=1}^{m} \delta t^{n} A_{D, \delta t}^{n}\left(u^{n}\right) \geq \int_{\Omega} \phi\left(\xi\left(\pi_{D} u^{m}\right)-\xi\left(\pi_{D} u^{0}\right)\right) d \mathbf{x} .
$$

For all $a \in \mathbb{R}$ it holds $\frac{1}{2} \underline{\xi} a^{2} \leq \xi(u) \leq c(a) a \leq \frac{(c(a))^{2}}{\bar{\xi}}$, therefore

$$
\sum_{n=1}^{m} \Delta t^{n} A_{D, \Delta t}^{n}\left(u^{n}\right) \geq \frac{1}{2} \underline{\xi \phi}\left\|\pi_{D} u^{m}\right\|_{L^{2}(\Omega)}^{2}-\frac{1}{\underline{\xi} \bar{\phi}}\left\|c\left(\pi_{D} u^{0}\right)\right\|_{L^{2}(\Omega)}^{2}
$$

Using the assumptions $\left(H_{2}\right)-\left(H_{3}\right)$ we deduce that $B_{D, \delta t}^{n}\left(u^{n}\right) \geq \underline{K}\left\|\nabla_{D} u^{n}\right\|_{L^{2}(\Omega)^{d}}^{2}$ and that $C_{D, \delta t}^{n}\left(u^{n}\right) \leq \overline{k_{r}} \bar{K}|\Omega|^{1 / 2}\left\|\nabla_{D} u^{n}\right\|_{L^{2}(\Omega)^{d}}$ for all $n \in\{1, \ldots, N\}$. Combining these inequalities with (9) and (11) gives 


$$
\begin{aligned}
\frac{1}{2} \underline{\xi} \underline{\phi}\left\|\pi_{D} u^{m}\right\|_{L^{2}(\Omega)}^{2}+\underline{K} \sum_{n=1}^{m} \delta t^{n}\left\|\nabla_{D} u^{n}\right\|_{L^{2}(\Omega)^{d}}^{2} \\
\leq \frac{1}{\underline{\xi} \bar{\phi}}\left\|c\left(\pi_{D} u^{0}\right)\right\|_{L^{2}(\Omega)}^{2}+\overline{k_{r}} \bar{K}|\Omega|^{1 / 2} \sum_{n=1}^{m} \delta t^{n}\left\|\nabla_{D} u^{n}\right\|_{L^{2}(\Omega)^{d}} .
\end{aligned}
$$

Applying Young's inequality to the last term above, we obtain

$$
\overline{k_{r}} \bar{K}|\Omega|^{1 / 2} \sum_{n=1}^{m} \delta t^{n}\left\|\nabla_{D} u^{n}\right\|_{L^{2}(\Omega)^{d}} \leq \frac{1}{2 \varepsilon}{\overline{k_{r}}}^{2} \bar{K} T|\Omega|+\frac{\varepsilon}{2} \bar{K} \sum_{n=1}^{m} \delta t^{n}\left\|\nabla_{D} u^{n}\right\|_{L^{2}(\Omega)^{d}}^{2} .
$$

This leads to

$$
\begin{gathered}
\frac{1}{2} \underline{\xi} \underline{\phi}\left\|\left(\pi_{D, \delta t} u\right)\right\|_{L^{\infty}\left(0, T ; L^{2}(\Omega)\right)}^{2}+\left(\underline{K}-\frac{\varepsilon}{2} \bar{K} \mid\right)\left\|\nabla_{D, \delta t} u\right\|_{L^{2}\left(Q_{T}\right)^{d}}^{2} \\
\leq \frac{1}{\underline{\xi} \bar{\phi}}\left\|c\left(\pi_{D} u^{0}\right)\right\|_{L^{2}(\Omega)}^{2}+\frac{1}{2 \varepsilon} \bar{k}_{r}^{2} \bar{K} T|\Omega| .
\end{gathered}
$$

One completes the proof of the estimate (7) by choosing $\varepsilon=\underline{K} / \bar{K}$ and using the assumptions $\left(H_{1}\right)$ and $\left(H_{4}\right)$.

The following result is rather standard and given without proof.

Proposition 2 Let $u$ be a solution to (6). There exists a positive constant $C$ only depending on $\underline{\phi}, \bar{\phi}, \underline{\xi}, \bar{\xi}, \underline{K}, \bar{K}, \overline{k_{r}}, \Omega, T, u_{0}, \hat{u}$ as well as on $\left\|c\left(\pi_{D} u^{0}\right)-c\left(u_{0}\right)\right\|_{L^{2}(\Omega)}$, $\left\|\pi_{D} \hat{u}_{D}-\hat{u}\right\|_{L^{2}(\Omega)}$ and $\left\|\nabla_{D} \hat{u}_{D}-\nabla \hat{u}\right\|_{L^{2}(\Omega)}$ such that for all $\tau \in(0, T)$, there holds

$$
\int_{0}^{T-\tau} \int_{\Omega}\left(\pi_{D, \delta t} u(\mathbf{x}, t+\tau)-\pi_{D, \delta t} u(\mathbf{x}, t)\right)^{2} d \mathbf{x} d t \leq C \tau
$$

Theorem 1. Let $\left(D^{(m)}, \delta t^{(m)}\right)_{m \in \mathbb{N}}$ be a family of discretizations, where $\left(D^{(m)}\right)_{m \in \mathbb{N}}$ assumed to be limit conforming, consistent, compact and uniformly coercive in the sense that there exist $C_{1}$ such that $C_{D^{(m)}} \leq C_{1}$ for all $m \in \mathbb{N}$; moreover we assume that $\left\|c\left(\pi_{D^{(m)}} u_{m}^{0}\right)-c\left(u_{0}\right)\right\|_{L^{2}(\Omega)},\left\|\pi_{D^{(m)}} \hat{u}_{D^{(m)}}-\hat{u}\right\|_{L^{2}(\Omega)}$ and $\left\|\nabla_{D^{(m)}} \hat{u}_{D^{(m)}}-\nabla \hat{u}\right\|_{L^{2}(\Omega)}$, $\max _{n} \delta t^{(m), n}$ tend to 0 as $m \rightarrow \infty$. Let $u_{m}$ be a solution of $(6)$ for all $m \in \mathbb{N}$. Then, up to a subsequence

$$
\begin{aligned}
& \pi_{D^{(m)}, \delta t^{(m)}} u_{m} \rightarrow \bar{u} \text { in } L^{2}\left(Q_{T}\right), \\
& \nabla_{D^{(m)}, \delta t^{(m)}} u_{m} \rightarrow \nabla \bar{u} \text { in } L^{2}\left(Q_{T}\right)^{d},
\end{aligned}
$$

where $\bar{u} \in L^{2}\left(0, T ; H^{1}(\Omega)\right)$ is a solution of (4).

Proof. Using the compactness and the uniform coercivity of the sequence $D^{(m)}$ as well as Propositions 1 and 2, we deduce from Fréchet-Kolmogorov theorem that the sequence $\left\{\pi_{D^{(m)}, \delta t^{(m)}} u_{m}-\pi_{D^{(m)}} \hat{u}_{D^{(m)}}\right\}$ is relatively compact in $L^{2}\left(Q_{T}\right)$. Therefore, we may extract a subsequence of $\left\{u_{m}\right\}$ (denoted again by $\left\{u_{m}\right\}$ ) such that $\pi_{D^{(m)}, \delta t^{(m)}} u_{m}$ converges to some $\bar{u} \in L^{2}\left(Q_{T}\right)$ strongly in $L^{2}\left(Q_{T}\right)$ and $\nabla_{D^{(m)}, \delta t^{(m)}} u_{m}$ is weakly convergent in $L^{2}\left(Q_{T}\right)$. It follows from Lemma 7.1 of [1] that the subsequence $u_{m}$ can also be chosen in such way that $c\left(\pi_{D^{(m)}, \delta t^{(m)}} u_{m}\right)$ and $k_{r}\left(c\left(\pi_{D^{(m)}, \delta t^{(m)}} u_{m}\right)\right)$ 
converge strongly in $L^{2}\left(Q_{T}\right)$ to $c(\bar{u})$ and $k_{r}(c(\bar{u}))$ respectively; moreover one deduces from (7) that $c(\bar{u}) \in L^{\infty}\left(0, T ; L^{2}(\Omega)\right)$. Finally we deduce from the limit conformity of the scheme that $\bar{u}-\hat{u} \in L^{2}\left(0, T ; H_{0}^{1}(\Omega)\right)$ and that $\nabla_{D^{(m)}, \delta t^{(m)}} u_{m} \rightarrow$ $\nabla \bar{u}$ in $L^{2}\left(Q_{T}\right)^{d}$ as $m \rightarrow+\infty$. Using again the limit conformity and consistency of the scheme we deduce that $\bar{u}$ is a weak solution of (4).

\section{Numerical tests}

\subsection{The Hornung-Messing problem}

The Hornung-Messing problem is a standard test (cf. for instance [5]). We consider a horizontal flow in a homogeneous ground $\Omega=[0,1]^{2}$ and set $T=1$. The problem after Kirchhoff's transformation is given by Problem (2) with

$$
c(u)=\theta(p)= \begin{cases}\pi^{2} / 2-2 \arctan ^{2}\left(\frac{u}{2-u}\right) & \text { if } p<0, \\ \pi^{2} / 2 & \text { otherwise },\end{cases}
$$

and suitable boundary and initial conditions. Let $s=x-z-t$, its solution is given:

$$
u(x, z, t)=\left\{\begin{array}{ll}
\frac{2 p(x, z, t)}{1+p(x, z, t)} & \text { if } p<0, \\
2 p(x, z, t) & \text { otherwise, }
\end{array} \quad p(x, z, t)= \begin{cases}-s / 2 & \text { if } s<0 \\
-\tan \left(\frac{e^{s}-1}{e^{s}+1}\right) & \text { otherwise }\end{cases}\right.
$$
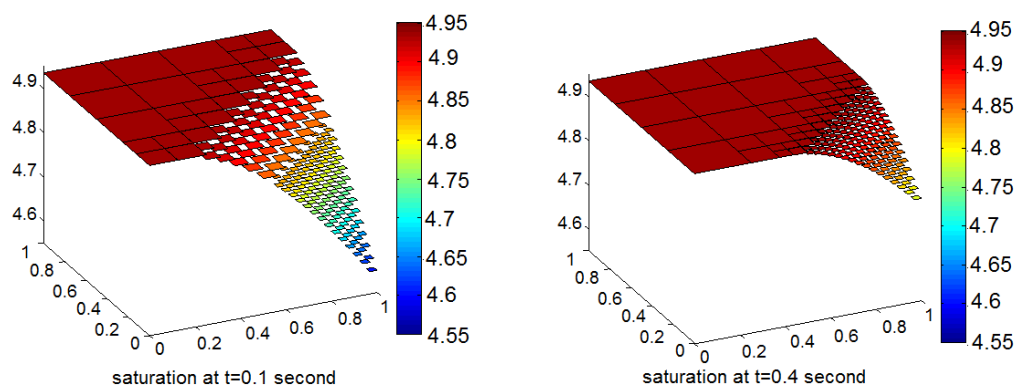

Fig. 1 Saturation at $t=0.1$ seconds and at $t=0.4$ seconds. The medium is unsaturated on the right-hand side of the space domain where $\theta<4.9348$ and fully saturated elsewhere.

In this test, we apply the Sushi scheme [4] using an adaptive mesh driven by the variations of the saturation. We prescribe the Neumann boundary condition deduced from (13) on the line $x=0$ and an inhomogeneous Dirichlet boundary condition elsewhere. We use an initially square mesh, which is such that each square can be 
decomposed again into four smaller square elements. Whereas the standard finite volume scheme is not suited to handle such a non-conforming adaptive mesh, the SUSHI scheme is compatible with these non-conforming volume elements.

We introduce the relative error in $L^{2}\left(Q_{T}\right)$ between the exact and the numerical solution as well as the experimental order of convergence

$$
\operatorname{err}(u)=\frac{\left\|\left(u_{\text {exact }}\left(\mathbf{x}, t_{n}\right)-u_{D, \delta t}\left(\mathbf{x}, t_{n}\right)\right)\right\|_{L^{2}\left(Q_{T}\right)}}{\left\|\left(u_{\text {exact }}\left(\mathbf{x}, t_{n}\right)\right)\right\|_{L^{2}\left(Q_{T}\right)}}, \quad \operatorname{eoc}=\frac{\log \left(\operatorname{err}\left(u_{i}\right) / \operatorname{err}\left(u_{i+1}\right)\right)}{\log \left(h_{D_{i}} / h_{D_{i+1}}\right)},
$$

where $u_{i}$ is the solution corresponding to the space discretization $D_{i}$. Table 1 shows the error using a uniform square mesh with various mesh sizes and time steps in the four first lines. Note that the scheme is only first order accurate with respect to time; therefore in order to obtain second order convergence we choose $\delta t$ proportional to $h_{D}^{2}$. We also compare the error for the approximate saturation using a uniform mesh and an adaptive mesh with a similar number of unknowns. In both cases: about 300 unknowns (line 2 - line 5) and 1200 unknowns (line 3 - line 6), the adaptive mesh compared to the fixed one provides slightly better results for the saturation $c(u)$. The observed computational gain is rather small (about $10-20 \%$ ), which is due to the fact that the area of high gradients of $c$ is comparatively large.

\begin{tabular}{|l|l|l|l|l|l|l|}
\hline Mesh & $N$ & $h_{D}$ & $N_{\text {unk }}$ & $\operatorname{err}(u)$ & $\operatorname{err}(c(u))$ & $\operatorname{eoc}(u)$ \\
\hline Uniform & 25 & 0.2 & 85 & $2.40 \cdot 10^{-2}$ & $1.60 \cdot 10^{-5}$ & - \\
\hline Uniform & 100 & 0.1 & 320 & $6.09 \cdot 10^{-3}$ & $4.13 \cdot 10^{-6}$ & 1.98 \\
\hline Uniform & 400 & 0.05 & 1240 & $1.53 \cdot 10^{-3}$ & $2.90 \cdot 10^{-6}$ & 2.00 \\
\hline Uniform & 1600 & 0.025 & 4880 & $3.76 \cdot 10^{-3}$ & $1.83 \cdot 10^{-6}$ & 2.02 \\
\hline Adaptive & 200 & 0.143 & 302 & $5.62 \cdot 10^{-3}$ & $3.67 \cdot 10^{-6}$ & - \\
\hline Adaptive & 800 & 0.071 & 1232 & $1.32 \cdot 10^{-3}$ & $2.19 \cdot 10^{-6}$ & - \\
\hline
\end{tabular}

Table 1 Number of time steps $N$, mesh diameter $h_{D}$, number of unknown $N_{u n k}$, the error of solution $\operatorname{err}(u)$, the saturation $\operatorname{err}(c(u))$ and the experimental order of convergence $e o c$.

\subsection{The Haverkamp problem}

We consider the case of a sand ground represented by the space domain $\Omega=(0,2) \times$ $(0,40)$ on the time interval $[0,600]$. The parameters are given by [7]

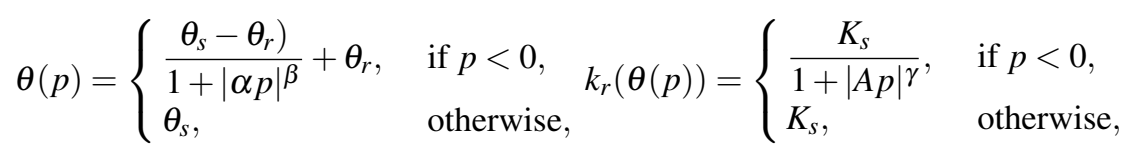

where $\theta_{s}=0.287, \theta_{r}=0.075, \alpha=0.0271 \beta=3.96, K_{s}=9.44 e-3, A=0.0524$ and $\gamma=4.74$. From $\theta$ and $K$, we have tabulated suitable values for the functions $c$ and 
$K_{c}$. We have taken here the initial condition $p=-61.5$, a homogeneous Neumann boundary condition for $x=0$ and $x=1$, the Dirichlet boundary condition $p=-61.5$ for $z=0$ and $p=-20.7$ for $z=40$.

We use an adaptive mesh and the time step $\delta t=1$ to perform a test. Figure 2-(a) represents the pressure profile at various times. In this test, no analytical solution is known. Therefore we compare our numerical solution with that of Pierre Sochala [8, Fig. 2.6, p. 35] which is obtained by means of a finite element method. Our results are quite similar to his. Figure 2-(b) shows the time evolution of the mesh at different times corresponding to the pressure profiles in Figure 2-(a).
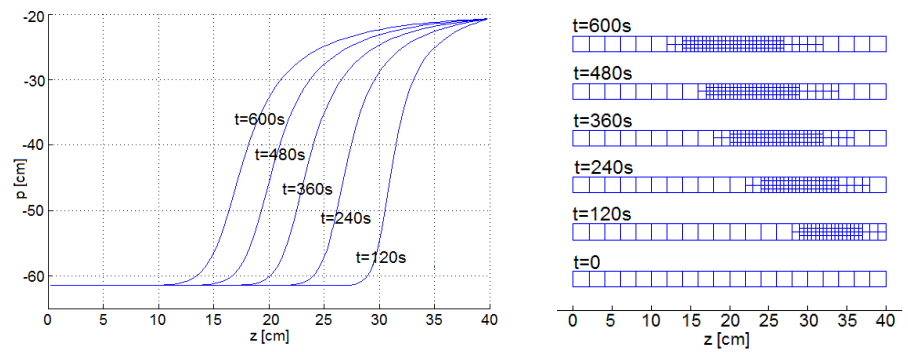

Fig. 2 Time evolution of the pressure $p$ and the adaptive mesh.

\section{References}

1. O. Angelini, K. Brenner D. Hilhorst. A finite volume method on general meshes for a degenerate parabolic convection-reaction-diffusion equation, Numer. Math. 123:219-257. DOI 10.1007/s00211-012-0485-5, 2013.

2. J. Droniou, R. Eymard, T. Gallouet, R. Herbin. Gradient schemes: a generic framework for the discretisation of linear, nonlinear and nonlocal elliptic and parabolic equations, Math. Models Methods Appl. Sci. 23(13), 2395-2432, 2013.

3. R. Eymard, C.Guichard, R. Herbin, R. Masson. Gradient schemes for two-phase flow in heterogeneous porous media and Richards equation ZAMM DOI:10.1002/zamm.201200206, 2013.

4. R. Eymard, T. Gallouët, R. Herbin. Discretization of heterogeneous and anisotropic diffusion problems on general nonconforming meshes. Sushi: a scheme using stabilization and hybrid interfaces, IMA J. Numer. Anal. 30(4): 1009-1043, 2010.

5. R. Eymard, M. Gutnic, D. Hilhorst. The finite volume method for Richards equation, Comput. Geosci. 3(3-4): 259-294, 2000.

6. R. Eymard, D. Hilhorst, M. Vohralik. A combined finite volume scheme nonconforming/mixedhybrid finite element scheme for degenerate parabolic problems. Numer. Math., 105:73-131, 2006.

7. R. Haverkamp, M. Vauclin, J. Touma, P. Wierenga, G. Vachaud. A Comparison of Numerical Simulation Models for One-Dimensional Infiltration. Soil Science Society of Am. Jour. 41.2:285294, 1977.

8. P. Sochala, Méthodes numériques pour les écoulements souterrains et couplage avec le ruissellement, Thèse de doctorat, Ecole Nationale des Ponts et Chaussées, 2008. 